\title{
Fundamentals of the philosophical content methodology on the creation and development of a class of natural and technical systems in water management construction
}

\author{
Vladimir Bondarenko ${ }^{1}$, Alla-Brerdi Ylyasov $^{2}$, Elena Semenova ${ }^{3}$, and Maria Shtavdaker ${ }^{1, *}$ \\ ${ }^{1}$ Novocherkassk Engineering And Land Reclamation Institute OfDon State Agrarian University, \\ 346428, Novocherkassk, Russia \\ ${ }^{2}$ WTL branch (USA) in Asia Innovators str., 7A Block. 2 Business Center "Navigator", Moscow, \\ Russia \\ ${ }^{3}$ Branch of the North Caucasus Federal University in Pyatigorsk, North Caucasian Federal University \\ (branch in Pyatigorsk), 357500, Pyatigorsk, Russia
}

\begin{abstract}
The main purpose of this article is to assess the philosophical, categorical concept meaning of the notions "system", "system approach" and "system research" in the new class of natural and technical systems (NTS) "Natural Environment - Activity Object- Population" ("NE - AO - P"), which have received theoretical and practical application in economic and other activities related to the use of water resources in almost all technological processes of industrial and agricultural production, in electric energy generation at hydroelectric power plants, pumped storage hydro stations, nuclear power plants, thermal power plants and regional power stations, in water supply of urban farms, settlements and other areas. In accordance with this concept in the considered branch of economic activity it is necessary to find a certain place of this concept in the general process of developing the natural-technical systems class theoretical foundations. One of the scientific thinking formations features in the creation of a new class of natural and technical systems is the wide distribution of system research related to the use of water resources in various sectors of economic activity. The concept of "system", "system research", "system approach", "system analysis" and other "system" concepts are widely used today, primarily in scientific and technical disciplines - general systems theory. They serve in these disciplines as an important conceptual basis for theoretical constructions and for the practical application of new scientific ideas and achievements. At the same time, the use of system concepts is becoming increasingly widespread in all previously established fields of science, including in water management science related to the use of water resources and technology. The concepts of " system "and" system approach " have already gained the status of general scientific and general technical concepts.
\end{abstract}

*Corresponding author: shtawkader.marya@yandex.ru 


\section{Introduction}

The processes of modern evolutionary development of society are characterized by the increasingly pronounced tendency to better understand the surrounding natural habitat, in which all life processes determining the multifaceted types of human economic activity, where a special type is economic activity associated with the use of water resources take place. That causes an objective need for the creation of new conceptual models of the natural and natural-technical environment.

The theoretical and methodological basis for the creation of new conceptual models of the natural and natural-technical environment in economic activities for the use of water resources is "system analysis" and "systems approach", as a general conceptual basis in the creation of a class of natural and technical systems «NE - AO - P».

"System analysis" and "systems approach" in the creation and development of the systems theory foundations (STF), is based on the central concept «System». Superiority in the use of this concept can be seen in Aristotle's works. The following basic ideas of STF are found in Hegel:

- The whole is more than the sum of the parts.

- The whole determines the nature of the parts.

- Parts cannot be cognized when viewed outside the whole.

The parts are in constant interconnection and interaction.

Explicit mass assimilation of system concepts, public awareness of the systemic nature in the world, society and economic activity began in 1948, when the American mathematician N. Wiener published a book called "Cybernetics".

Water management science associated with the use of water resources is one of the studied systems' variants, where natural and technical systems are investigated. In these systems the "Natural environment" and water management facilities, referred to as "Activity Objects" («AO»), and "Population", living in «AO» influence zones are considered.

In systemic studies of the considered class of natural and technical systems, the following basic concepts are used: "system", "systems approach", "systems analysis", "systems research" and other systemic concepts that are widely used at the present stage of the science - general systems theory (GST) development, bionics, information theory, factor analysis, systems engineering and others. These systemic concepts act as the most important concentration basis in theoretical constructions and for the practical application of new scientific ideas and achievements. At the same time, the use of system concepts is becoming more and more widespread in water management science, related to the use of water resources with ensuring environmental safety. The concepts "system" and "systems approach" won the general scientific and general technical concepts status and, accordingly, received such a status in water science, which contributes to the development of the most important branch of economic activity related to the use of water resources.

As the modern process of concepts development shows, that "element" and "structure" in the considered class of natural and technical systems appeared somewhat earlier than the concept "system", received the status of general scientific and general technical and represent the first empirical step towards their transformation into new philosophical categories. The final, "theoretical" step on this path was the philosophical generalization of these concepts. Based on dialectical-materialistic analysis of "element" and "structure" concepts development, this generalization made it possible to reveal that the basis of their widespread use in various scientific and technical fields is the presence of a certain internal unity, essential commonality in all their various variations. This community turned out to be an extremely abstract theoretical reflection of such universal sides and connections, relations of the material world, which were not fully covered by previously formed philosophical categories. So, there was an objective basis for the inclusion of general scientific concepts of 
"element" and "structure" into the existing system of philosophical categories. These concepts joined this system, found a definite place in it as regularly arising the new stages of cognitive, theoretical, methodological, practical assimilation of reality in creating a class of natural and technical systems for water resources use in economic.

\section{Materials and methods}

In connection with the transformation of the concept "system" in general scientific and general technical, the task of philosophical generalization of this concept arises, identifying its specifically philosophical content, checking the possibility of considering it as a new philosophical category in relation to the class of natural and technical systems. The fact is that philosophical categories, performing a general methodological function in the knowledge of the material world, reflect the universal aspects and relationships, connections between the real objects in a certain historical and logical sequence. In general, it coincides with the sequence in which the universal aspects and relations of reality, fixed in philosophical, extremely general concepts, that is, categories, became dialectical and logical forms of thinking in the course of the historical knowledge development in the world on the basis of its practical transformations.

At the level of philosophical reflection of reality, this development includes, first of all, a natural change, the enrichment of the already established philosophical categories' content. But besides this, the development of philosophical cognition in reality also turns out to be a process of the natural emergence (on the basis of objective logic) of new dialectical and logical categories. This process takes place when, at new stages of social development, the theoretical and practical assimilation of reality with the help of new concepts becomes a necessary condition for scientific and technological progress, the improvement of material production and other areas of social practice, including the use of water resources in various industries.

The main purpose of this analysis is to try to identify the philosophical, categorical meaning of the concept "system" and in accordance with this meaning, to find a certain place of this concept in the general process of creating and developing a class of natural and technical systems.

\section{Results}

Categorical meaning of the concept "system". To establish the categorical meaning of the concept of "system", it is necessary to single out a common, single point - invariant meaning in various senses and uses of this concept, then find out which general aspects and connections of the material world are reflected in this general moment. The key to understanding the specifics of the latter in the case of a philosophical generalization of the concept "system" there should be a study of the question why this concept, formulated initially in general in a rather special area of theoretical knowledge of the world - in the sphere that is the subject of the general theory of systems created by L. Bertalanffy [3] - has spread further to all the most important areas of modern science and technology.

This could happen only because the concept formulated in the framework of the general theory of systems, nevertheless, reflected a certain essential aspect of understanding objects of any nature as systems. Only because of this, this concept turned out to be applicable, with one or another variation, to all those objects that, in the course of knowledge and practice development, should have been considered by the researchers as systemic objects, for example, water management objects within basin geosystems. This suggests that the categorical content of the concept "system" is largely inherent in a more or less explicit form 
in various concepts of the system, developed by various researchers, including the class of natural and technical systems.

The definition of a system given by L. Bertalanffy as a complex of interacting elements is essentially applicable to any objects and is still the basis of all (existing both within the framework of the general theory of systems and outside these frameworks) "system" concepts. Actually, even before, any researcher most often knew that the objects studied by him consist of parts of components (elements). On the whole, however, science has been predominantly concerned with the isolated study of these parts. The study of their joint action remained in the background of scientific knowledge. Ashby correctly notes that the strategy of scientific research has mainly been the analysis and dismemberment of a complex whole into simple parts so far [6]. V.N. Sadovsky also rightly points out that the specific features that distinguish the "systems approach" from other types and forms of scientific knowledge are associated with the rejection of such an approach "... from one-sided analytical, linearcausal research methods..." [7].

The emphasis on the integrity and integration of the objects' features, made in defining the system as a complex of interacting components and their constituent elements, was precisely the basis for its widespread distribution, including in water science. At present, science is increasingly reaching such a level of its development when the study of the joint action of parts as part of a whole comes to the fore, when the theoretical development of such objects, the nature of which does not allow ignoring the effects of interaction between components and their constituent elements, begins, when finally As S. Young correctly thinks [8], the researchers were forced to start creating such complex systems that the most important task in creating new design solutions taking into account environmental requirements was the task of ensuring integrity by integrating parts into a single one. An important role in the formation of a new approach was played by the concept of a system, which arose both in the framework of the general theory of systems and in the methodology of creating a new class of natural and technical systems.

What is this role? Having made a special emphasis not on the fact that the whole consists of parts, but on the fact that the behavior and features of the whole are determined by the interrelation, interaction and interrelation of its parts, that the whole consists of interacting parts, the concept "system" becomes the basis of a new, predominantly synthetic view of the objects under study, which is most strongly expressed in the systemic concept of scientific knowledge. One of the most important aspects of a systemic study of a class of natural and technical systems, without any doubt, is to consider them as a complex of interrelated and interacting components and their constituent elements, which ensures success in creating a generalized methodology and is connected precisely with such a consideration of water management objects of cognition, placing special emphasis on the interaction of components and their constituent elements as the reason for the presence of features in the whole that are not available in its components and, accordingly, elements. The general theory of systems, as well as the methodology of natural-technical systems, agree that the solution to the issue to consider or not to consider some entity consisting of parts as a system - depends on whether we set the task of studying the behavior of parts and their interactions [9].

However, the systemic approach is not limited with the approach to the considered object as a complex of interacting parts (elements). Thus, V.N. Sadovsky and E.G. Yudin, trying to isolate some invariant of the concept "system", consider the interconnection of the components and their constituent elements of the system only as one of the variants of this invariant. Other options from their point of view are: a system that forms a special unity with the environment, and as a rule, any system is a part of a system of a higher hierarchical order; part of the system under consideration, in turn, act as systems of a lower order [10].

The fact that the system forms a special unity with the environment acts as the next really fundamental moment of the concept "system": to study an object as a system means to study 
its interaction with the environment, to have the subject of research: its changes as an integral formation in response to certain environmental influences. Thus, in addition to considering an object as a complex of interacting components and their constituent elements, the second main point of systemic research is its focus on studying the behavior of an object in its environment. Therefore, the definition of a system as a complex of interrelated and interacting components among themselves leaves the essence of a systemic study: a naturaltechnical system is always studied as a part of another system higher in the hierarchical row, covering this one as its component, and such a study of it is aimed at understanding the features of its behavior as a result interaction of its own components and their constituent elements. An object, for example a reservoir, is considered in a systematic study as a complex of interacting natural, technogenic components and the living population precisely in order to study the behavior of this object in the corresponding environment as an integral formation.

Due to this, a natural-technical system is not only a certain whole, made up of certain interacting components, but such a set of components that has a certain behavior as a part of a higher hierarchical row, more complex system - the surrounding natural environment. This concept of a system directs the researcher to the fact that nothing else is studied but the behavior of an object as an integral formation in a systemic study and it is studied precisely from the point of view of the internal structure of the object, from the point of view of its components' interaction, that is, the task systemic research is to explain the features of the behavior and the features of an integral object, based on the specifics of its components and the features of their interaction with each other. Implicitly, this, of course, implies both Bertalanffy's definition and, apparently, all other definitions. Consequently, it is precisely the moments highlighted that constitute, perhaps, the most general basis for any variations of the concept "system". Therefore, their unification into a single concept in order to highlight the categorical meaning of the concept "system" in the considered class of natural and technical systems, perhaps, makes it possible to get a general scientific and general methodological concept "system" and "systems research". This integral concept contains the most general methodological prescriptions regarding what and how is studied in any systemic study, for example, assessment of water management facility impact on the natural environment (AWMIE).

Any material formation is in constant interaction with other material formations is changed in this interaction, that is, it becomes in a motion state. Cognition can be directed at a given moment only at the movement of material education, its changes, that is, a person can study how material education moves, without studying how what is moving. Such knowledge is a study related to the material formation behavior sphere, for example, a reservoir that regulates water flow (surface, underground) in the spatial limits of the basin geosystem.

But a person can have anything that moves as the object of his cognition, temporarily leaving without consideration the question of how the cognized thing moves. Such knowledge is associated with the material education structure sphere study. Thus, what a researcher can turn into an object of cognition refers either to the sphere of material formation behavior, or to the sphere of its structure, or to connection, the unity of behavior and structure, which consists primarily in the conditioning of behavior by the structure.

Behavior is one of the opposite sides of an object as a system: the side associated with movement, with the change of this object, with its interaction with other objects, which in their totality make up the environment of the studied object-system. Movement, all kinds of changes in the object under study are the results of its relationship, interaction and interconnection with the natural environment in the considered spatial limits of the basin geosystem, where water resources are formed.

In the class of natural and technical systems, changes in a water management object are the result of its interaction with natural environments, as well as the result of the interaction 
of its internal components and their elements with each other. Cognition of these results, of course, is one of the most important points in the study of the studied water management object's behavior. A water management object appears in the knowledge as a system only if its interaction is studied both with natural environments and with other objects located higher or lower in the hierarchical row of the basin geosystems. Therefore, the study of the water management object-system behavior includes the knowledge of both the changes occurring with it and those interactions, i.e., the reasons that caused these changes. Thus, from an epistemological point of view, the concept "behavior" reflects the relationship between changes in material education and their causal influences.

For this concept to be a really effective "tool" of system research, it is necessary to clarify it as follows. Knowledge of the water management object behavior does not require, as it may seem at first glance, an exhaustive study of the interactions that have caused a certain change in the water management object. To consider the behavior of a material formation (and not just its changes), but at the same time not to be drawn into an exhaustive study of interaction as the ultimate cause of changes, it is enough to find a connection between the individual changes and those factors of interactions that turned out to be direct causal effects on water management of the object that generated the corresponding changes. Thus, the "systems approach" to a water management object (precisely because it is associated with the consideration of its behavior as one of the two opposite sides objectively inherent in the object) is a way of studying this object as a system that transforms direct causal influences into appropriate changes. This transformation of influences on the system into its changes is the real, ontological behavior of the considered class of natural and technical systems.

The effectiveness of such notion as "systems research" lies primarily in the fact that it reflects such an important aspect of material formations in the form of water management objects, which allows the quantitative study and mathematical description. Based on the knowledge of the entire set of correspondences that exist between the changes in the water management object studied by the researcher and the impacts that caused these changes, the researcher is able to identify the functional relationships between certain subsets of the impacts on the water management object and its changes. Having realized this opportunity, the researcher will be able to describe the behavior of a water-related object in the form of a set of functions.

In a philosophical sense "behavior" is a concept that fixes such universal moments of objective reality as a change in material formations, direct causal influences that cause these changes, and the connection between them. The behavior of material education is objectively a unity of two opposite sides: changes and immediate causal influences. Category "behavior" reflects the objective connection of these opposite sides, that is, it turns out to be, in a sense, a synthesis of such opposite categories as "behavior" and "consequence", synthesis, the urgent need for which arises at the stage of scientific knowledge, when the approach to the object as to "system".

The structure of a material formation as a side opposite to behavior, in turn, turns out to be a unity of opposites: a set of elements and a set of connections between them - a structure that integrates the components and their elements into some holistic formation. In the light of this, it becomes clear that the currently widespread definitions "system" how the set of interacting, interconnected or interdependent components and their constituent elements actually fix only such a moment of the categorical meaning of the concept "system", as a structure, or construction of a system. Like the category "behavior", category "structure", reflecting the objective unity of such opposite sides of material education as components and structure, it turns out to be a synthesis of opposite categories "component" and "structure", synthesis, the urgent need for which arises in connection with the deployment of systemic studies of the natural and technical systems' class. 
In turn, the category "system" turns out to be a synthesis of opposite categories "behavior" and "structure", synthesis, reflecting such universal moments of objective reality as the presence of each material entity, on the one hand, behavior (i.e., the transformation of causal influences on it into corresponding changes in its state, carried out by any water management object), on the other hand, material the bearer of behavior (that is, structure, the specificity of which determines the characteristics of the behavior of a given material formation); finally, on the third hand, the existence of an objective connection "behavior" with "structure". The reflection of this connection is the essence of the categorical meaning of the concept "system". The latter, as a dialectical-logical category, is a reflection of any material formation from the point of view of the unity of its behavior and structure, a reflection of such a universal connection between "behavior" and "structure", as the conditioning of the behavior of this whole by the specifics of its internal structure, the specifics of its elements and the features of the interaction between them. Consequently, any water-economic object appears in cognition as "system" only if the researcher considers it not just as a set of interconnected, structure-integrated components and their constituent elements - a structure, but as a structure that transforms causal influences from the surrounding natural environment, and from within the system into corresponding changes to the water management facility as a whole.

The logical need for a category "system" as a stage in the creation and development of a class of natural and technical systems. The categorical meaning of the concept "system", is determined by the need to use it in class creation NTS «NE - AO - P» in scientific knowledge. We considered this necessity mainly only in connection with the formation of the systems class general theory foundations. To fully characterize the general scientific concept "system" as a new philosophical category is possible only if the logical-historical necessity of its transformation into a dialectical-logical category is revealed, i.e., the latter is derived from logic and the knowledge development history, and it is presented with other philosophical categories as a natural stages of knowledge development. This process of category deduction "system" from logic and the history of cognition, as a result of which it will become clear what place it should occupy in the system of dialectic categories, will at the same time be the process of proving the categorical meaning of the concept identified above "system". The merit of this proof will be that it is carried out from the point of view of objective logic and the history of cognition, from the point of view of what content the concept should have "system" as a necessary step in knowledge development in creating a class of natural and technical systems "Natural Environment - Activity Object - Population".

The process of cognition begins with the isolation of individual material formations, things and processes from the infinite variety of reality, with the understanding of the individual as a universal form of the existence of matter, that is, with the reflection of the real world through the category "separate". At the next stage of cognition, a connection is established between individual material formations. Therefore, the categories "separate" and "connection" represent the first, initial stage of knowledge development and practice $[2,3]$. Moreover, the initial stage of the knowledge and practice development, these categories turn out to be both in logic and in the history of the development of cognitive and practical activities for people.

But since the objective "decomposition" of the water management object of cognition into such aspects as behavior and structure turns out to be limited of all those moments of the object that can become the subject of cognition, insofar as cognition proceeding from a certain idea of the object as a separate, undivided whole connected with other separate wholes, begins its deepening into the object under study either from a separate moment related to the sphere of "behavior" of the object, or from a separate moment of the sphere of its "buildings". Thus, studying a water management object in the form of a separate, undivided whole, cognition immediately "dismembers" a separate whole into separate parts 
related to one of its indicated opposite sides. Logically, cognition of an object, as a separate part, is directed further to "behavior" this separate, because it fixes, first of all, the movement of the considered water-economic object, its changes.

Cognition "behavior" of a separate material formation then develops as a certain logicalhistorical process.

Cognition "behavior" is to reveal the correspondence between the changes in the water management facility and the causal influences that caused these changes. Therefore, at the very initial stages, it is carried out as a qualitative and quantitative study of individual changes, followed by the presentation of changes as functions of causal influences, that is, a description of behavior by a variety of functions. Knowledge of these functions already makes it possible to predict some features of the water management facility behavior under certain causal influences. To achieve precisely this goal, a functional, mathematical description of the behavior of a water management facility is carried out. At the same time, the predictive power of the functional description of behavior lies not only in the fact that, by substituting some possible influences into the corresponding functions, we obtain knowledge about the possible behavior of a water management object. This predictive power is also associated with the fact that some of the functions of the object behavior mathematical description make it possible to calculate a number of its properties, which, along with the functions, are also capable of characterizing the "behavior" of a water management object and knowledge of which allows predicting many features of behavior within the geosystems' basin.

In addition to the functional calculation, the source of knowledge about some features of the water management object can be directly served by the experimental data on its "behavior", changes, phenomena. Cognition of the set of functions that describe the behavior of a given object, and the totality of the "functional (that is, deduced by a functional calculation)" and experimental (that is, derived directly from the experimental data) features of a water-economic object is the main content of the first - functional - the stage of cognition "behavior" water management object, and at the same time the entire investigated water management object within the spatial limits of the basin geosystem. The ultimate goal of this stage of cognition is to establish general, stable, necessary correlations between causal influences and changes in a water management facility, i.e., the disclosure of functional laws "behavior" - the laws of functioning of a given object, its functional essence, which is the so-called essence of the first order.

If the object was studied for the purpose of managing its "behavior", then the need for its further study may not arise, because this goal, within fairly wide boundaries, can be achieved precisely on the basis of knowledge of the functions and functional laws that describe "behavior" water management facility. However, the functional stage of a water management object cognition is the study of only its "behavior" — does not allow to give answers to the following questions: why the behavior of a given object is exactly what it is; why the water management facility has exactly the established, and not any other functional and experimental properties; why finally, "behavior" the water facility is governed by the data, and not by any other functional laws. The formulation of these questions, obviously, constitutes the limit of the functional stage of a water management object cognition, because the study of only the behavior itself, its study, as such, without referring to the specifics of the structure, object, does not allow to overcome this limit.

Since the functions and features that characterize "behavior" a water management object is nothing more than the functions and features of its specific structure, insofar as the disclosure of the structure of this object turns out to be the ultimate goal of knowing its "behavior", finite in the sense that the explanation of behavior and functional laws on the basis of the features of the structure as a material carrier of this "behavior" represents the highest type of explanation of the facts of behavior, movement, changes in the object under 
consideration. It is possible to know how a water management facility functions, what changes it responds to causal influences, even without knowing its structure, but to understand why it functions the way it functions, it is possible only by revealing its specifics "structure". The explanation of any phenomena, facts of behavior, properties, functions, functional laws for any material formations consists, ultimately, in their derivation from the specifics of the components and their constituent elements and structure of material formations. This position is one of the central methodological principles of all modern natural science, and it increasingly penetrates into the methodology of water management sciences, in particular, the study of the natural and technical systems class.

The fact that cognition "behavior" material education in the form of a natural-technical system, sooner or later, should logically end with the study of the structure of this education, it turns out to be necessary (not only logically, but also historically) for the most profound and relatively complete cognition, first of all "behavior", but at the same time, and with respect to a wide study of the entire water management facility, exhaustive in the sense that (as a result of the transition from studying "behavior" to knowledge "structure" a given object) two objectively inherent aspects of it - behavior and structure - turn out to be cognized and connected in the structural and functional methodology, reflecting the essential features "behavior" water management facility and giving a structural explanation of the features of this "behavior".

Consequently, human knowledge of any separate material formation develops logically as follows: it starts with the involvement of this formation in the sphere of the cognitive (as well as practical) activity of the subject as a separate undivided whole, then it is directed towards isolation and becomes the study of one of its opposite sides: "behavior" as logically the whole is the first in the study of the individual; finally, in the logical sense, the knowledge of the object turns into a study and the opposite "behavior" side of the object - its "structure". Ultimately, the logic of the knowledge development of a separate material formation, the latter appears before the researcher as a unity "behavior" and "structure", that is, as a system - a separate holistic formation, but already divided into its relatively cognized opposite sides and studied further from the point of view of the interconnection, interaction and relationship of these parties, their interdependence with each other.

Thus, cognition, starting from the separate, again returns to the separate. But, having made a spiral ascent along a number of steps from the separate as an undivided whole, cognition again returns to the separate already as to "system", dismembered into "behavior" and "structure". The task of studying a separate material formation at the point from which knowledge is just beginning to develop and which is characterized by categories "separate" and "connection", is to completely exhaust the possibilities of purely functional knowledge of this water management object, separate in connection with other separate ones, and thus relatively complete the study of its "behavior" in interconnection, interaction and relationship with natural environments without any reference to its material carrier. The next point of the cognition movement, which is, in essence, a dialectical negation of the original, has its goal the study of this carrier and thus the coverage of both opposite sides of the separate. As a result of this coverage, cognition again, as it were, returns to the starting point of its development - to the separate (but to the separate - already at a new stage of theoretical mastery of the object). The task of cognizing the individual in this new starting point, at a new turn in the spiral of developing knowledge, becomes the study of unity, interconnection "behavior" and "structure", further, deeper and more detailed study of the class of natural and technical systems. 


\section{Conclusion}

In the creation and development of a class of natural and technical systems, the concept "System" is considered as the relationship, interaction and interconnection between natural (biotic, abiotic), man-made (water management facility) and social (residing population) components within the spatial limits of the basin geosystem, where quantitative and qualitative indicators of water resources are formed in the form of surface and ground water runoff.

The main method in the creation and study of a class of natural and technical systems is "systems approach", in which the system as a whole is considered, and for improving and functional efficiency to ensure the environmental safety of its constituent components with their constituent elements, are considered as subsystems. "Systems approach" in the class of natural and technical systems is used as a design methodology, as a conceptual basis in the development of a class of natural and technical systems.

\section{References}

1. L. Bertalanffy, General theory of systems - critical review. Research on the theory of systems (Moscow, 1969).

2. V.L. Bondarenko, G.M. Skibin, V.N. Azarov, E.A. Semenova, V.V. Privalenko Environmental safety in environmental engineering, water use and construction: assessment of the ecological state of the basin geosystem: Monograph South-Russian State Polytechnic University (NPI) named after M.I. Platov (SRSPU (NPI), Novocherkassk, 2016).

3. V.L. Bondarenko, E.A. Semenova, A.V. Aliferov, O.V. Klimenko, Natural and technical systems in the use of water resources: basin geosystem territory: monograph (SRSPU(NPI), Novocherkassk, 2016).

4. R. Dzhonson, F. Kast, D. Rozenczvejg, Systems and guidance (Moscow, 1971).

5. I. Klir, The abstract concept of a system as a methodological tool. Research in General System Theory (Moscow, 1969).

6. M.V. Kovalchuk, From synthesis in science to convergence in education, Educational Policy 11-12, 49-50 (2010).

7. M.V. Kovalchuk, O.S. Narajkin, Constructor for the future, In the world of science 9, 24-31 (2011).

8. Nikolaj Kuzanskij, book series Philosophical heritage 1, (Moscow, 1979), 2. (Moscow, 1980).

9. G. Nikolis, I. Prigozhin, Knowledge of the complex (Mir, Moscow, 1990).

10. I. Prigozhin, I. Stengers, Order out of chaos (Progress, Moscow, 1986).

11. A. M. Chernyaeva, River basins, RosN IIVX. Ekaterinburg (Agricultural Ecology, 1999).

12. V.N. Sadovskij, E.G. Yudin, Tasks, methods and provisions of the general theory of the system (Research in General System Theory, Moscow, 1969).

13. S.N. Smirnov, Some features of modern scientific and technological progress, Forecasting social processes in a socialist society 1, Kiev, 1969.

14. Federal Law of January 10/2002 № 7-FL « About environmental safety and protection»

15. U.R. Eshbi, General systems theory as a new scientific discipline (Research in General System Theory, Moscow, 1969). 
16. S. Yang, System organization management (Moscow, 1972).

17. L. Bertalanffy, An outline of General System Theory, British Journal for the Philosophy of Science 1, 1950. 